\title{
Regionalisierung hydrologischer Modelle mit Function Space Optimization
}

\author{
Moritz Feigl (iD - Mathew Herrnegger (D) - Robert Schweppe $($ D $\cdot$ Stephan Thober - Daniel Klotz (D) \\ Luis Samaniego (iD) $\cdot$ Karsten Schulz (iD
}

Angenommen: 15. April 2021 / Online publiziert: 17. Mai 2021

(C) Der/die Autor(en) 2021, korrigierte Publikation 2021

Zusammenfassung Das Schätzen von räumlich verteilten Parametern hydrologischer Modelle ist ein bereits lang erforschtes und anspruchsvolles Problem. Parameter-Transferfunktionen, die einen funktionellen Zusammenhang zwischen Modellparametern und geophysikalischen Gebietseigenschaften herstellen, sind eine potenzielle Möglichkeit, Parameter ohne Kalibrierung zu schätzen. Function Space Optimization (FSO) ist eine symbolische Regressionsmethode, die automatisiert Transferfunktionen aus Daten schätzen kann. Sie basiert auf einem textgenerierenden neuronalen Netzwerk, das die Suche nach einer optimalen Funktion in ein kontinuierliches Optimierungsproblem umwandelt.

In diesem Beitrag beschreiben wir die Funktionsweise von FSO und geben ein Beispiel der Anwendung mit dem mesoscale Hydrological Model (mHM). Ziel der Anwendung ist die Schätzung zweier Transferfunktionen für die Para-

The original online version of this article was revised: co-author Luis Samaniego was missing

DI M. Feigl ( $₫)$ · DI Dr. M. Herrnegger . Univ.-Prof. Dipl.-Geoökol. Dr. K. Schulz Institut für Hydrologie und Wasserwirtschaft, Universität für Bodenkultur Wien

Muthgasse 18, 1190 Wien, Österreich moritz.feigl@boku.ac.at

R. Schweppe, MSc. · Dr. S. Thober .

Dr. L. Samaniego

Department

Hydrosystemmodellierung,

UFZ-Helmholtz Zentrum

für Umweltforschung,

Permoserstraße 15, 04318 Leipzig,

Deutschland

DI Dr D. Klotz

LIT AI Lab \& Institute für Machine Learning, Johannes Kepler Universität Linz, Altenberger Straße 69, 4040 Linz, Österreich meter KSat (gesättigte hydraulische Leitfähigkeit) und FieldCap (Feldkapazität). Dafür verwenden wir Daten 7 großer deutscher Einzugsgebieten über einen Zeitraum von 5 Jahren zum Schätzen der Transferfunktionen und weiterer numerischer Parameter. Die resultierenden Funktionen und Parameter werden ohne weitere Kalibrierung auf 222 Validierungsgebiete über eine Validierungsperiode von 35 Jahren angewendet. Mit der Anwendung in diesen „unbeobachteten“ Gebieten können wir die Übertragbarkeit und die zumindest regionale Gültigkeit der Transferfunktionen überprüfen.

Die Ergebnisse zeigen, dass bei einer Anwendung in unbeobachteten Gebieten die Modellgüte in einem ähnlichen Wertebereich wie in den Trainingsgebieten liegt und somit weiterhin akzeptabel ist. Die Nash-Sutcliffe Efficiency (NSE) in den Trainingsgebieten über den Validierungszeitraum unterscheidet sich mit einem medianen Wert von 0,73 nicht nennenswert von dem der Validierungsgebiete mit einem medianen NSE von 0,65 .

Zusammengefasst haben Transferfunktionen das Potenzial, die Vorhersagefähigkeiten, Übertragbarkeit auf andere Gebiete sowie physikalische Interpretierbarkeit bestehender hydrologischer Modelle zu verbessern. Mit FSO wurde zum ersten Mal eine objektive, datengetriebene Methode entwickelt, mit der Transferfunktionen geschätzt werden können.

Schlüsselwörter Hydrologische Modellierung

Parameterregionalisierung $\cdot$ Deep

Learning · Vorhersage in

unbeobachteten Einzugsgebieten

\section{Regionalization of hydrological models using function space optimization}

Abstract The estimation of distributed hydrological model parameters is a long studied and challenging problem. Parameter transfer function, i.e. mathematical equations that describe the relationship between model parameters and geo-physical catchment properties, make it possible to potentially estimate parameters without the need for calibration. Function Space Optimization (FSO) is a symbolic regression method for automatically estimating parameter transfer functions from data. FSO is based on a text generating neural network that transforms the search for a best fitting transfer function into a continuous optimization problem.

In this contribution we discuss FSO and its characteristics and show its applicability with the mesoscale Hydrological Model (mHM). The aim of this application is the estimation of transfer functions for two model parameters: KSat (saturated hydraulic conductivity) and FieldCap (field capacity). We use the data of 7 large German Basins and 5 years of data to estimate the transfer functions and additional numerical parameters of mHM. The resulting functions und parameters are applied without any further calibration to 222 validation basins with 35 different years of data. By applying the model in these "ungauged" basins, it is possible to estimate the transferability in time and space of the estimated transfer functions.

The application in ungauged basins result in model performance values which are in similar range compared to the training basins. The median NashSutcliffe Efficiency (NSE) in the training basins in the validation time-period is 0.73 , while the median NSE of the validation basins in the same time period is 0.65 .

Transfer functions have the potential to increase the performance, the transferability, and the physical interpretability of the model parameters of existing hydrological models. FSO is the first objective, data-driven method for 
estimating parameter transfer functions for hydrological models.

Keywords Hydrological modelling Parameter regionalization - Deep Learning · Predictions in ungauged basins

\section{Einleitung}

Verteilte hydrologische Modelle sind ein weit verbreitetes Werkzeug zur Modellierung räumlich und zeitlich verteilter Prozesse in Einzugsgebieten. Dazu gehört die Modellierung räumlich verteilter Austauschprozesse zwischen Landoberfläche und Atmosphäre (e.g. Rakovec et al. 2016), der hydrologischen Änderungen durch den Klimawandel (e.g. Hattermann et al. 2017; Kay et al. 2015) oder hydrologischer Änderungen durch Landnutzungsänderungen (e.g. Hundecha und Bárdossy 2004; Wijesekara et al. 2012). Grundsätzlich können prozessbasierte, verteilte hydrologische Modelle in zwei Gruppen eingeteilt werden: konzeptuelle Modelle und physikalisch-basierte Modelle (Devia und Ganasri 2015). Beide benötigen bis zu einem gewissen Ausmaß eine Parameterkalibrierung (Beven 2001; Kirchner 2006), was dazu führt, dass in beiden Fällen umfangreiche Expertise im Bereich der Modellkalibrierung benötigt wird. Während konzeptionelle Modelle per Definition eine Parameterkalibrierung benötigen, fehlen physikalisch-basierten Modellen häufig die nötigen Messungen, um alle notwendigen Modellparameter zu definieren. Gleichzeitig ist es mit den derzeit vorhandenen Messmethoden nicht möglich, die hohe 3-dimensionale räumliche Heterogenität der die hydrologischen Prozesse beeinflussenden geophysikalischen Einzugsgebietseigenschaften zu messen. Noch herausfordernder und unrealistischer erscheint dabei die flächige Erfassung der Eigenschaften im Untergrund, da hier zusätzliche satellitengestützte Fernerkundungsmethoden keine Rückschlüsse ziehen lassen, zumindest nicht in einer räumlichen Auflösung, die für eine Modellanwendung relevant wäre. In der Praxis führt das dazu, dass die Parameter daher häufig als physikalische Konstanten definiert werden (Clark et al. 2017), oder - wie bei konzeptionellen Modellen in Rahmen einer Parameterkalibrierung optimiert oder „skaliert“ werden. Beide Ansätze resultieren in einer Reduktion des Prozessrealismus, während sie trotzdem realistische Abflussvorhersagen produzieren.

Eine mögliche Lösung zur Erhaltung des Prozessrealismus hydrologischer Modelle wäre, einen Zusammenhang zwischen Landschaftseigenschaften und hydrologischen Prozessen zu definieren (Clark et al. 2016). Das könnte durch die Definition der Modellparameter auf Basis geophysikalischer Information erreicht werden. Dies ist jedoch nicht trivial, weswegen Clark et al. (2017) es als eines der großen ungelösten Probleme der hydrologischen Parameterkalibrierung sieht. Erst kürzlich erwähnten auch Blöschl et al. (2019) die „Entwirrung und Reduktion der Struktur/Parameter/Input-Unsicherheiten für hydrologische Modelle“ als eines der dreiundzwanzig wichtigsten ungelösten Probleme der Hydrologie. Modellparameter, die durch geophysikalische Eigenschaften eines Einzugsgebiets definiert werden, würden Parameterunsicherheiten reduzieren, Prozessrealismus und Vorhersagefähigkeit und Übertragbarkeit des Modells verbessern sowie die Vorhersage in Einzugsgebieten ohne Abflussdaten ermöglichen.

Dieses Problem ist eng verwandt mit der Idee der Regionalisierung, also der regionalen Übertragung hydrologischer Modellstrukturen (Buytaert und Beven 2009). Aufgrund des Problems der Parameter-Äquifinalität hydrologischer Modelle (Beven 2006), d.h., dass unterschiedliche Parametersätze zu gleich guten Ergebnissen führen, führt die Suche nach einem Zusammenhang nach einer Parameterkalibrierung zu einer schwachen oder falschen Regionalisierung (Hundecha und Bárdossy 2004; Kumar et al. 2013a; Samaniego et al. 2010). Um das zu vermeiden, wurde die simultane Regionalisierung (Abdulla und Lettenmaier 1997; Hundecha und Bárdossy 2004; Parajka et al. 2005) entwickelt. Dabei wird im Vorhinein ein Zusammenhang zwischen Modellparametern und geophysikalischen Gebietseigenschaften in Form einer Transferfunktion definiert und an mehreren Validierungseinzugsgebieten getestet.

Samaniego et al. (2010) entwickelten aufbauend auf der Idee der simultanen Regionalisierung die „multiscale parameter regionalization (MPR)“Methode. Bei MPR werden Parameter mit Transferfunktionen auf der jeweils kleinstskaligen Auflösung der Gebietseigenschaften berechnet, bevor sie auf die Modellauflösung aggregiert werden. Dabei wird der Informationsverlust der kleinskaligen Informationen der Gebietseigenschaften deutlich verringert, die physikalische Interpretation der Parameter bleibt erhalten und es werden kontinuierliche Parameterfelder erzeugt, welche nicht die in verteilten Modellen häufig auftretenden Unstetigkeiten, z.B. an Einzugsgebietsgrenzen, aufweisen (Samaniego et al. 2017). Dadurch wird die Übertragbarkeit der Modelle und deren Parametrisierung deutlich verbessert, weswegen Modelle mit MPR in Gebieten ohne Abflussmessungen zum Einsatz kommen können.

Anstatt (wie häufig üblich) Transferfunktionen mithilfe eines Regressionsansatzes $\mathrm{zu}$ definieren, sind alle Transferfunktionen in MPR klar definierte mathematische Funktionen der geophysikalischen Gebietseigenschaften. Diese mathematischen Funktionen sind jedoch meist unbekannt, weshalb die größte Herausforderung bei der Anwendung von MPR die Definition passender Parametertransferfunktionen ist (Samaniego et al. 2017). Mögliche Kandidaten für hydrologische Modelle könnten Pedo-Transferfunktionen sein, welche den Zusammenhang zwischen Bodenparametern und Bodeneigenschaften beschreiben und bereits intensiv untersucht wurden (siehe z.B. Van Looy et al. 2017). Der funktionelle Zusammenhang zwischen Modellparametern und geophysikalischen Gebietseigenschaften ist allerdings kaum bekannt und es gibt bisher auch keine Methoden, um diese (objektiv) zu schätzen.

Ein möglicher Ansatz, Transferfunktionen für MPR $\mathrm{zu}$ finden, ist die Symbolische Regression (Koza 1992). Der Ausdruck „Symbolische Regression" beschreibt Methoden, die den Raum der mathematischen Funktionen durchsuchen, um ein Fehlermaß zu minimieren. Diese Methoden basieren häufig auf evolutionären Algorithmen (Bongard und Lipson 2007; Cornforth und Lipson 2015; Schmidt und Lipson 2009). Klotz et al. (2017) und Klotz (2020) zeigten mithilfe eines simplen hydrologischen Modells und synthetischen Daten, dass es grundsätzlich möglich ist, Transferfunktionen mit Symbolischer Regression zu optimieren. Aufbauend darauf entwickelten Feigl et al. (2020) die „Function Space Optimization (FSO)“. FSO überführt die Suche nach einer besten Transferfunktion in ein kontinuierliches Optimie- 
rungsproblem mithilfe eines neuronalen Netzwerks. Dabei handelt es sich um ein textgenerierendes Netzwerk, das trainiert wurde, um mathematische Funktionen („Text“ im weitesten Sinne) aus numerischen Vektoren $\mathrm{zu}$ generieren. Damit wird die Suche nach einer besten Transferfunktion zu einem konventionellen kontinuierlichen Optimierungsproblem, wie es in der Hydrologie häufig auftritt und mit dem es schon viel Erfahrung gibt.

Ziel dieses Artikels ist es, einen Überblick über die „Function Space Optimization“ als nützliche Methode im Bereich der Modellparameter-Regionalisierung zu geben und ihre Funktionalität anhand eines Beispiels zu zeigen. Weiters geben wir einen Ausblick auf zukünftige Entwicklungen und weiteren Anwendungsmöglichkeiten in der Hydrologie.

\section{Material und Methoden}

\subsection{Transferfunktionen für die} automatisierte Optimierung

Bei (Parameter-)Transferfunktionen handelt es sich um mathematische Gleichungen, die den Zusammenhang zwischen Modellparametern und Einzugsgebietseigenschaften beschreiben. Dabei kann es sich um simple lineare Funktionen in Abhängigkeit von einer Variablen handeln, z. B.

\section{Parameter $=1.3+$ Sandgehalt $\cdot 0.2$,}

aber auch - theoretisch - hochgradig nicht-lineare Funktionen multipler Variablen, z. B.

$$
\begin{gathered}
\text { Parameter }=\frac{\exp \left(\text { Hangneigung }^{3}\right)+2}{\sqrt{-\log (\text { Sandgehalt })}} \\
+\sinh (\text { Höhe }) \frac{\text { atan }(\text { Bodendichte })}{\sqrt{\text { Tongehalt }}}
\end{gathered}
$$

Des Weiteren kann auch eine beliebige Anzahl an numerischen Koeffizienten vorhanden sein, die theoretisch beliebig groß sein können. Da wir in den meisten Fällen keinerlei Vorwissen über die Struktur und die Koeffizienten der zugrundeliegenden „wahren“ Transferfunktion haben, muss die Suche möglichst offen gestaltet werden.

Ein Problem, das dabei auftritt, ist der unterschiedliche Wertebereich der möglichen geophysikalischen Variablen (z. B. Höhe, Sandgehalt, Neigung, Bodendichte, Leaf Area Index). Bei der manuellen Suche nach Transferfunk- tionen kann dieses Problem mithilfe passend gewählter numerischer Koeffizienten ausgeglichen werden (siehe z.B. Samaniego et al. 2010). Bei der automatischen Suche würde man aber eine starke Gewichtung der Variablen mit höheren Werten erzeugen sowie einen großen Wertebereich der Koeffizienten benötigen. Beides würde die objektive Suche nach einer passenden Transferfunktion erschweren.

Um das Wertebereich-Problem $\mathrm{zu}$ lösen, werden für die automatische Transferfunktionsoptimierung alle Variablen mittels einer min-max-Skalierungsfunktion auf den Wertebereich [0, 1] skaliert:

$$
x_{[a, b]}=a+\frac{(x-\min (x))(b-a)}{\max (x)-\min (x)},
$$

D.h., eine Variable $x$ wird auf das Intervall $[a, b]$ skaliert, wobei wir zunächst $\mathrm{a}=0$ und $\mathrm{b}=1$ wählen. Das benötigte Minimum/Maximum wird aber nicht aus den vorhandenen Daten geschätzt, sondern mithilfe physikalisch realistischer Grenzen definiert, z. B. Sandgehalt $\in[0,100]$. Dadurch können die gefundenen Transferfunktionen in jedem anderen Einzugsgebiet angewendet werden.

Um die mit einer Transferfunktion generierten Werte in den Wertebereich des jeweiligen Parameters zu skalieren, wird zur Berechnung der endgültigen Parameterwerte noch einmal die minmax-Skalierungsfunktion angewendet. Das Zielintervall ist dabei durch die physikalisch sinnvollen und realistischen Parametergrenzen im hydrologischen Modell definiert. Minimum und Maximum der Transferfunktionen hingegen beziehen sich nicht auf die Werte der jeweiligen Transferfunktion, sondern auf alle für das Training vorhandenen Transferfunktionen. Eine grafische Darstellung einer beispielhaften Skalie- rungsabfolge für Transferfunktionen ist in Abb. 1 ersichtlich.

Als zweiter wichtiger Punkt für die Implementierung einer automatisierten Suche nach geeigneten Transferfunktionen ist die Definition eines Suchraums - also der möglichen Strukturen und Variablen, die eine Funktion definieren können. Dafür verwenden wir eine kontextfreie Grammatik (contextfree Grammar, CFG) (Knuth 1965). Diese besteht aus Variablen, Operatoren und strukturellen Regeln, mit denen Text erzeugt werden kann. Ein simples Beispiel einer CFG ist in Abb. 2 dargestellt.

CFGs bestehen aus „Nonterminals“, denen unterschiedliche "Mappings“ mit möglichen Werten zugeordnet sind. Sie können wie ein Entscheidungsbaum gelesen werden, bei dem man beim ersten Nonterminal beginnt und jeweils ein Mapping auswählt, bis keine Nonterminals in der Funktion mehr vorhanden sind. Damit kann mit einfachen Regeln eine große Anzahl an möglichen Funktionen definiert werden. Vor allem durch das Verwenden rekursiver Mappings kann die Komplexität stark erhöht werden.

Mit diesen zwei Konzepten, Skalierungsabläufe für Transferfunktionen und CFGs zum Erzeugen von Transferfunktionen, sind die Grundlagen für die automatisierte Suche nach Transferfunktionen definiert. Sie bilden die Basis für die im nächsten Schritt folgende Optimierungsmethode.

\subsection{Function Space Optimization (FSO)}

FSO wurde entwickelt, um die Suche nach einer passenden Transferfunktion in ein objektives Optimierungsproblem umzuwandeln. Die initiale Idee von FSO basiert auf der Interpretation von mathematischen Gleichungen als Text

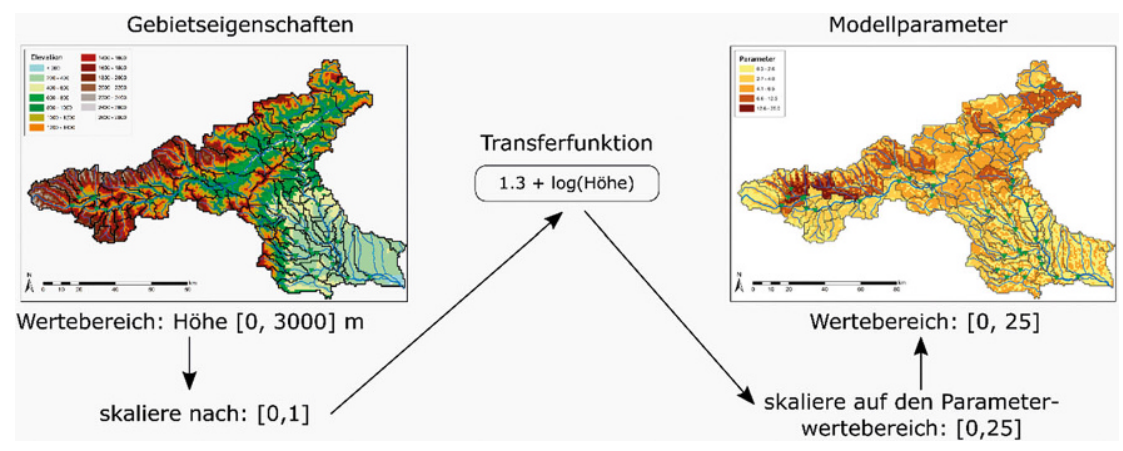

Abb. 1 Beispiel für die Skalierungsabfolge für die automatisierte Suche nach optimaIen Transferfunktionen. Beide Skalierungsschritte verwenden die min-max-Skalierung 


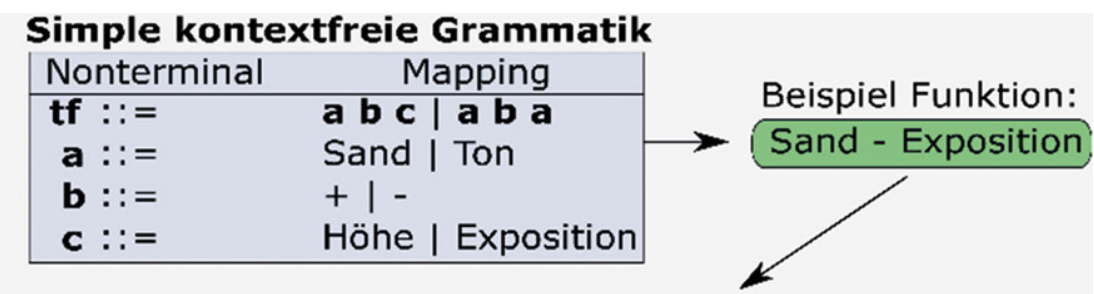

Funktion als Pfade eines Entscheidungsbaumes

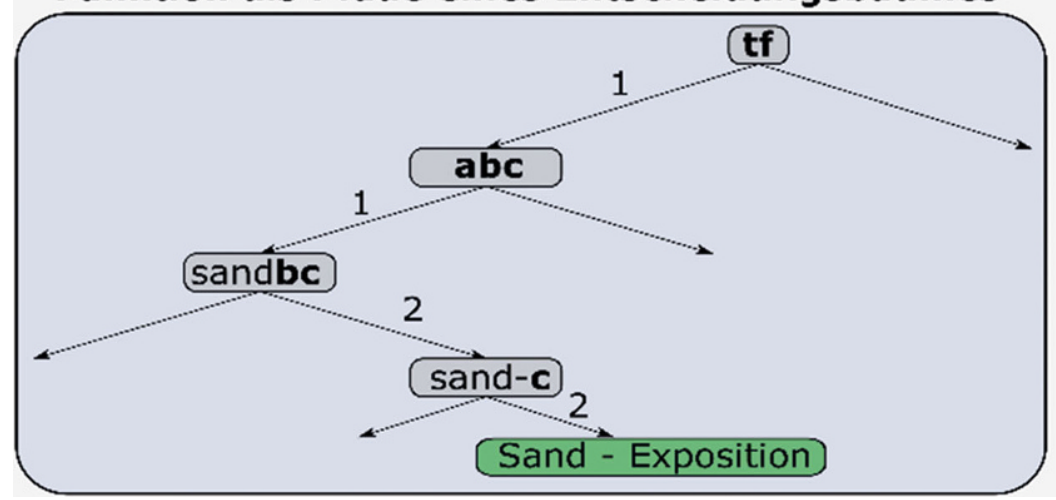

Abb. 2 Beispiel einer simplen kontextfreien Grammatik (CFG)

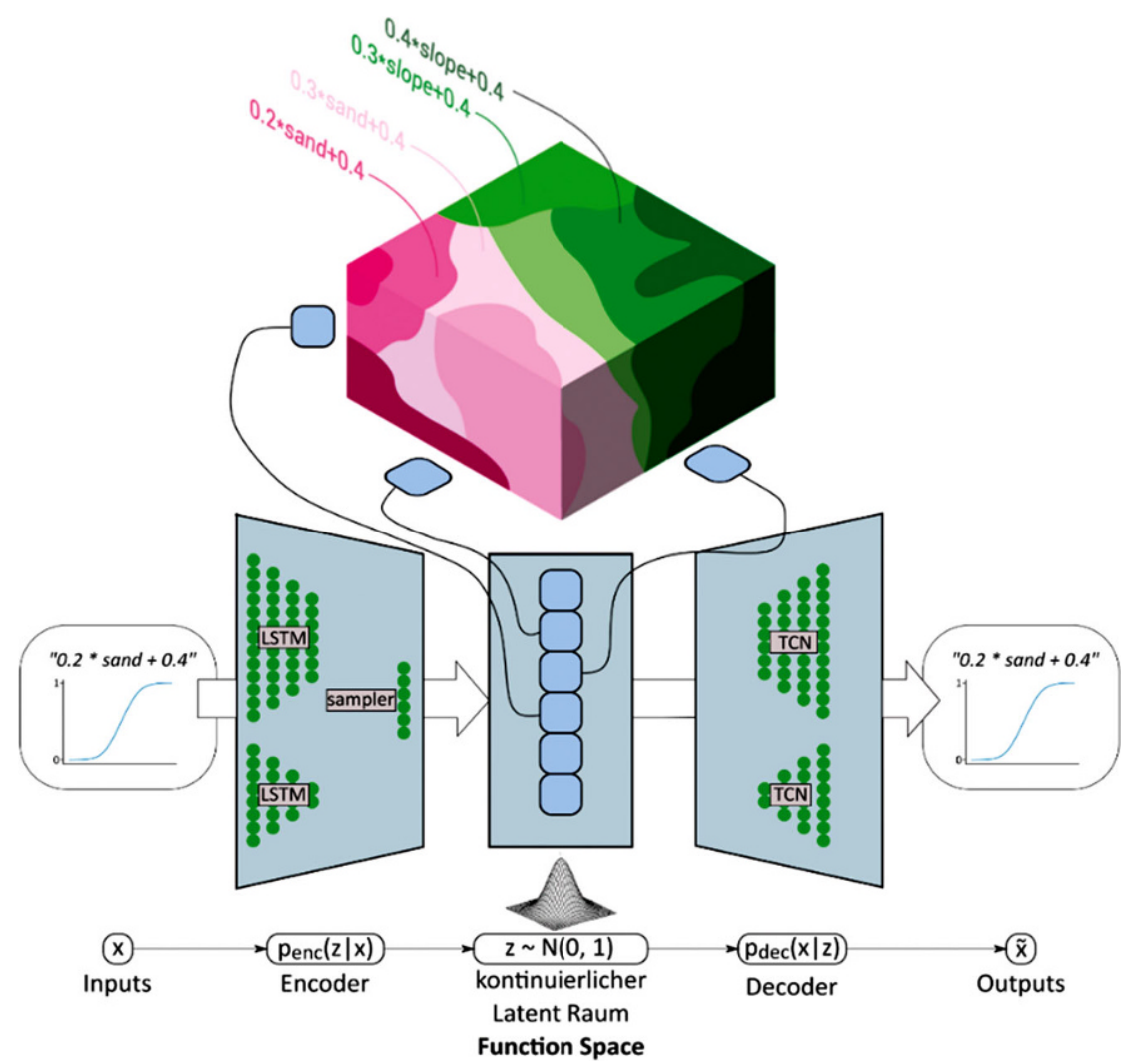

Abb. 3 Schematische Darstellung des FSO Variational Autoencoder. Er besteht aus einem Encoder- und einem Decoder-Netzwerk. Der Encoder komprimiert die Information der Transferfunktionen und ihrer Verteilungsfunktionen (Quantile) in den Function Space. Der Decoder stellt die ursprüngliche Funktion und Verteilungsfunktion (Quantile) aus dem Function Space wieder her und den darin verwendeten Symbolen (Variablen, Operatoren, Funktionen, numerische Werte) als Wörter. Diese Interpretation ermöglicht es, Methoden aus der Computerlinguistik (engl.: Natural Language Processing) anzuwenden, welche in den letzten Jahren große Fortschritte im Bereich der Übersetzung (z. B. Srivastava et al. 2018), der Textgenerierung (z.B. Lu et al. 2018) und Textklassifizierung (z.B. Yang et al. 2019) gemacht hat.

Eine häufig angewendete Netzwerkarchitektur für textgenerierende Netzwerke ist die Autoencoder-Architektur (Le Cun und Fogelman-Soulié 1987). Dabei handelt es sich um ein aus zwei Teilen zusammengesetztes Netzwerk, einem Encoder und einem Decoder. Der Encoder erzeugt eine komprimierte Repräsentation der Inputs: den latenten Raum. Der Decoder rekonstruiert die Inputs aus der Repräsentation im latenten Raum. Der große Vorteil eines Autoencoders ist genau diese Projizierung in den latenten Raum, wodurch jegliche Input-Information in numerische Parameter komprimiert werden kann. In unserem Fall handelt es sich bei den Inputs um die Information der Transferfunktionen. Nach dem Trainieren des Autoencoders kann der Decoder verwendet werden, um aus beliebigen Positionen im latenten Raum Funktionen zu generieren. Genau das machen wir uns zunutze, um aus der Suche nach einer Transferfunktion eine kontinuierliche Optimierung im latenten Raum zu machen.

Da wir den latenten Raum für eine Optimierung verwenden wollen, ist es weiter sinnvoll, ihn einzuschränken bzw. ihm Charakteristiken zu geben, die eine Optimierung erleichtern. Dabei handelt es sich um drei spezifische Eigenschaften: (i) Der latente Raum sollte eine kompakte Repräsentation des Suchraums sein, (ii) die Distanz im latenten Raum soll semantische Ähnlichkeit von Funktionen wiedergeben, und (iii) die Distanz im latenten Raum soll die Ähnlichkeit der resultierenden Parameterverteilung der Transferfunktionen wiedergeben.

Um Eigenschaft (i) zu erfüllen, verwendet FSO eine Variational Autoencoder (VAE)-Architektur (Kingma und Welling 2013). Diese Weiterentwicklung des Autoencoders fördert durch einen zusätzlichen Fehlerterm und einen stochastischen sampling Layer im Encoder eine Standardnormalverteilung im latenten Raum. Eigenschaft (ii) und 
(iii) ergeben sich durch die Wahl der Inputs, einerseits die Transferfunktionen als Text, andererseits die 0,1-0,9Quantile ihrer resultierenden Parameterwerte. Eigenschaft (iii) ist notwendig, da durch Korrelation der geophysikalischen Eigenschaften (z.B. Sandund Tongehalt) unterschiedliche Funktionen sehr ähnliche Parameterfelder erzeugen können. Durch (i) bis (iii) erhalten wir einen kontinuierlichen Optimierungsraum, der das Suchen von Transferfunktionen erleichtert.

Eine schematische Darstellung der VAE-Struktur ist in Abb. 3 ersichtlich. Sowohl Encoder als auch Decoder sind durch neuronale Netzwerke abgebildet. Technisch gesehen ist der Encoder eine Kombination von Word Embedding (Mikolov et al. 2013), bidirectional long short-term memory (LSTM) network (Hochreiter und Schmidhuber 1997) und feedforward neural network (FNN) Layern (White und Rosenblatt 1963) mit SELU (scaled exponential linear unit) Aktivierungsfunktion (Klambauer et al. 2017), und der Decoder eine Kombination aus Temporal Convolutional Network (TCN) Layers (Bai et al. 2018) und FNN Layers mit softmax-Aktivierungsfunktion. Der FSO VAE komprimiert Information der Transferfunktionen in einen 6-dimensionalen latenten Raum, den wir „Function Space“ nennen.

Zum Trainieren des VAE werden Transferfunktionen aus einer vorher definierten CFG generiert und die Quantile ihrer Verteilungsfunktion abgeschätzt. Nach dem Training ist der VAEDecoder imstande, Transferfunktionen aus ihrer Darstellung im latenten Raum („Function Space“) wiederherzustellen. Daher ist es möglich, eine Optimierung im latenten Raum durchzuführen, um eine Transferfunktion zu schätzen. Der Decoder ist jedoch nicht nur imstande, Funktionen wiederherzustellen, sondern auch neue Funktionen zu generieren, die den allgemeinen Regeln der verwendeten CFG unterliegen. Demnach können nicht nur Funktionen, die im Trainingsdatensetz vorhanden waren, erzeugt werden, sondern auch andere Funktionen, mit den in der CFG vorgegebenen Strukturen.

FSO ist eine kontinuierliche Optimierungsschleife, bei der Transferfunktionen, aber auch andere numerische Parameter eines Modells optimiert werden können. Die von FSO generierten Transferfunktionen benötigen die im vorherigen Abschnitt beschriebene Skalierungsabfolge für eine unverzerrte

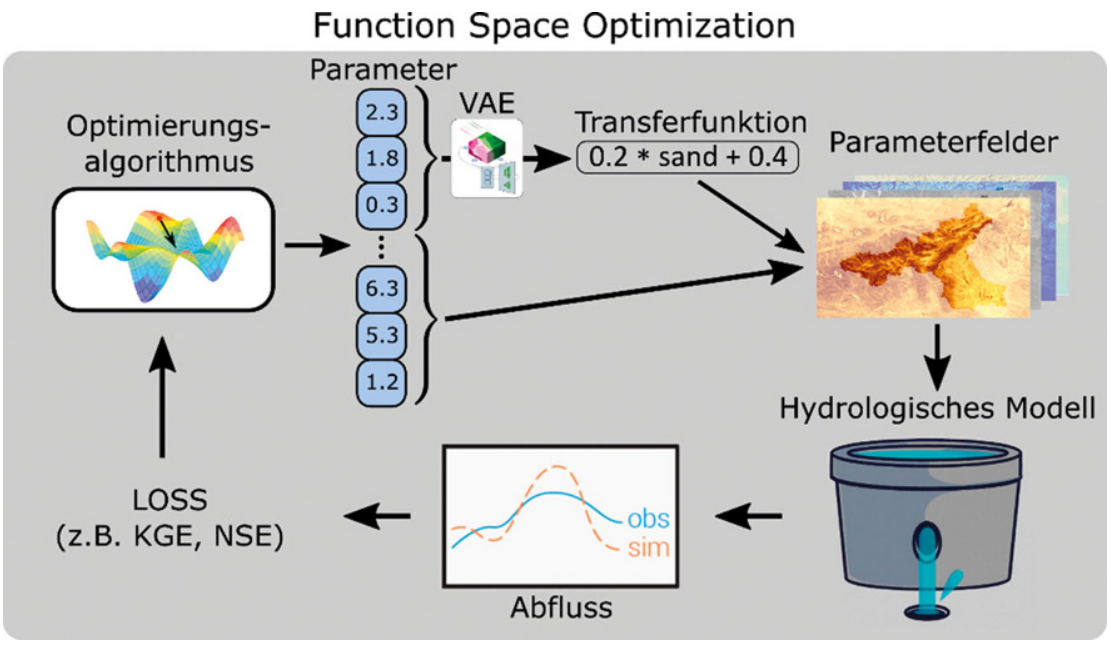

Abb. 4 FSO-Optimierungsschleife, bestehend aus einem Optimierungsalgorithmus, der numerische Parameter optimiert. Diese werden zum Erzeugen von Transferfunktionen sowie als numerische Parameter des Modells verwendet, um die Parameterfelder für das hydrologische Modell zuerzeugen. Mitdem vorhergesagten Abfluss des Modells kann ein Loss berechnet werden, welcher vom Optimierungsalgorithmus verwendet wird, um die Parameter der nächsten Iteration zu wählen

Optimierung. Jeglicher kontinuierlicher Optimierungsalgorithmus kann für FSO verwendet werden. Getestet wurden bisher der Genetische Algorithmus (Holland 1975), Dynamically Dimensioned Search (Tolson und Shoemaker 2007), Particle Swarm Optimization (Kennedy und Eberhart 1995) und der Shuffled Complex Evolution Algorithmus (Duan et al. 1992). Eine Darstellung der FSO-Optimierungsschleife ist in Abb. 4 zu sehen.

Die Zielfunktion der Optimierung (Loss-Funktion) für FSO kann beliebig gewählt werden und ist abhängig von der jeweiligen Zielsetzung eines Projekts, z. B. Nash-Sutcliffe Efficiency (NSE, Nash und Sutcliffe 1970) oder Kling-Gupta efficiency (KGE, Gupta et al. 2009). Grundsätzlich ist FSO für die Anwendung in mehreren Einzugsgebieten gedacht, da globale, oder zumindest regional anwendbare Transferfunktionen gesucht werden. Dementsprechend wird eine Transferfunktion gesucht, die in allen Einzugsgebieten gute Ergebnisse produziert. Um das zu erreichen, verwendet FSO immer ein gewichtetes Mittel aller Loss-Funktionen der einzelnen Einzugsgebiete:

$$
\operatorname{Loss}_{\mathrm{FSO}}=\frac{\sum_{i=1}^{I} w_{i} \operatorname{Loss}\left(Q_{i}, \widehat{Q}_{i}\right)}{\sum_{i=1}^{I} w_{i}},
$$

wobei Loss die gewählte Loss Funktion ist, $Q_{i}$ und $\widehat{Q}_{i}$ die beobachteten und vorhergesagten Abflüsse des Einzugsge- bietes $i$ sind, $w_{i}$ das jeweilige Gewicht des Einzugsgebietes $i$ ist und $I$ die gesamt Anzahl der Einzugsgebiete ist. Dabei werden alle Gewichte definiert durch $w_{i}=1-\operatorname{Loss}\left(Q_{i}, \widehat{Q}_{i}\right)$, unter der Annahme, dass die Loss Funktion ein mögliches Maximum von 1 hat. Damit ist der FSO-Loss ein gewichtetes Mittel, bei dem mehr Gewicht auf den Loss der Einzugsgebiete mit einem schlechteren Ergebnis gelegt wird.

\subsection{Beispielprojekt: mesoscale Hydrological Model (mHM)}

Dieser Abschnitt gibt einen Überblick über die Anwendung von FSO mit dem mesoscale Hydrological Model (mHM, Kumar et al. 2013b; Samaniego et al. 2010, 2019; Thober et al. 2019). mHM (www.ufz.de $/ \mathrm{mhm}$ ) ist ein verteiltes hydrologisches Modell, das auf der MPR-Methode basiert und Prozesse in mehrschichtigen Rasterzellen simuliert. Demnach sind alle Parameter mittels Transferfunktionen definiert, die Samaniego et al. (2010) mithilfe von Literaturwerten, einer „step-wise“-Methode (Samaniego und Bárdossy 2005) und „trial-and-error" hergeleitet haben. Die mHM-Transferfunktionen beinhalten numerische Koeffizienten, mit denen das Modell auf die jeweilige Region kalibriert werden kann. Die von mHM verwendeten numerischen Approximationen und Konzeptualisierungen basieren auf dem HBV-Modell (Bergström 


\begin{tabular}{|c|c|c|c|c|c|c|c|c|c|}
\hline \multirow[t]{2}{*}{ EZG } & \multirow[t]{2}{*}{ Fläche $\left(\mathrm{km}^{2}\right)$} & \multicolumn{2}{|c|}{ Höhe (m) } & \multicolumn{3}{|c|}{ Landnutzung (\%) } & \multicolumn{3}{|c|}{ Wasserbilanz $\left(\mathrm{mm} \mathrm{a}^{-1}\right)$} \\
\hline & & Mittel & SD & Wald & Versiegelt & Gemischt & P & $Q$ & Ea \\
\hline Mulde & 6200 & 386 & 201 & 26 & 10 & 64 & 798 & 344 & 454 \\
\hline Ems & 8400 & 60 & 36 & 13 & 8 & 79 & 802 & 312 & 490 \\
\hline Neckar & 12.700 & 445 & 153 & 35 & 10 & 55 & 914 & 356 & 558 \\
\hline Main & 23.700 & 356 & 113 & 39 & 6 & 55 & 793 & 247 & 546 \\
\hline Saale & 24.800 & 287 & 162 & 23 & 8 & 69 & 645 & 161 & 484 \\
\hline Weser & 37.700 & 223 & 165 & 34 & 7 & 59 & 781 & 276 & 505 \\
\hline Donau & 47.500 & 558 & 170 & 32 & 6 & 62 & 948 & 469 & 479 \\
\hline
\end{tabular}

1995) und beinhalten die Prozesse Interzeption, Schneeakkumulierung und Schneeschmelze, Infiltration und Oberflächenabfluss, Bodenwasserretention und Abflussgenerierung, Evapotranspiration, Perkolation, Basisabfluss und Routing. Eine detaillierte Beschreibung von mHM kann in Samaniego et al. (2010) gefunden werden.

Ziel dieser Anwendung ist die Generierung zweier neuer Transferfunktionen für die mHM-Parameter KSat (gesättigte hydraulische Leitfähigkeit) und FieldCap (Feldkapazität) mittels FSO. Höllering et al. (2018) zeigten, dass diese beiden mHM-Parameter hoch sensitiv sind und durch ihre Rolle bei der Parametrisierung des Speichers und der Leitfähigkeit des Bodenwassers einen wichtigen Beitrag zur Abfluss-

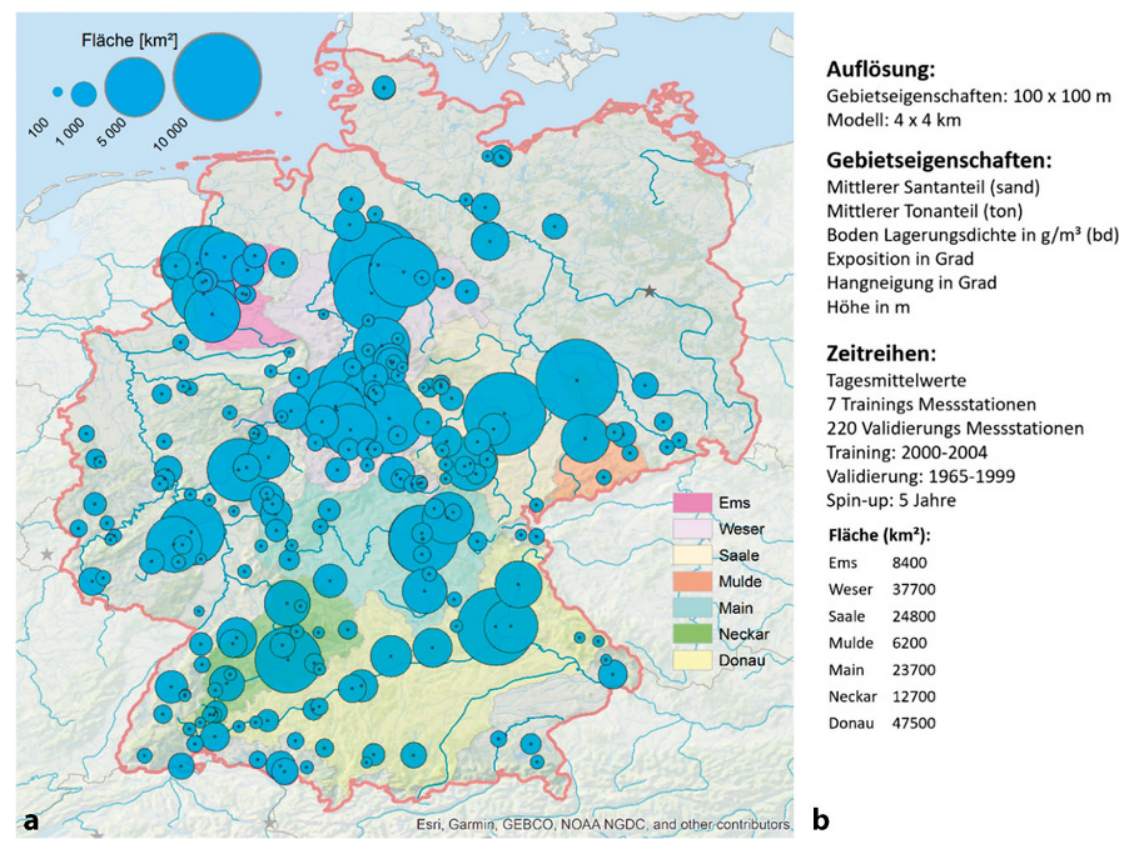

Abb. 5 a Übersicht der Fallstudiengebiete. Die Trainingsgebiete sind farblich hinterlegt und die Validierungsgebiete mit den Punkten ihrer Messstellen dargestellt, wobei die Punktgröße die jeweilige Einzugsgebietsfläche widerspiegelt. b Eine Übersicht des Modellaufbaus und der Gebietseigenschaften für die mHM-Optimierung mittels FSO wobei ThetaS der gesättigte Bodenwassergehalt, $\gamma_{-} 5$ und $\gamma_{-} 6$ numerische Koeffizienten und vGenu_n der vanGenuchten-Modellparameter (van Genuchten 1980) ist. Der Wert der mHMFeldkapazität ist demnach ein Anteil des gesättigten Bodenwassergehalts.

Zur Optimierung der Transferfunktionen und der Schätzung ihrer Anwendbarkeit in anderen Einzugsgebieten definieren wir zwei Gruppen von Einzugsgebieten: (i) Trainingsgebiete, die zur Suche der Transferfunktionen und Optimierung der restlichen mHM-Parameter verwendet werden, und (ii) Validierungsgebiete, die zum Testen der gefundenen Funktionen dienen. Dabei ist anzumerken, dass in den Validierungsgebieten keinerlei Art von Kalibrierung oder Optimierung stattfindet und nur die in den Trainingsgebieten gefunden Transferfunktionen und numerische mHM-Parameter angewendet werden. Um die Übertragbarkeit der gefunden Funktionen noch robuster schätzen $\mathrm{zu}$ können, werden für Trainings- und Validierungsgebiete unterschiedliche Zeitperioden simuliert.

Die zugrundeliegenden Daten und daher auch die Einteilung der Einzugsgebiete und Zeitperioden wurden von Zink et al. (2017) übernommen. Die Trainingsgebiete bestehen aus 7 sehr großen Einzugsgebieten in Deutschland. Es handelt sich dabei um die Flüsse Ems, Weser, Saale, Mulde, Main, Neckar und Donau. Die Validierungsgebiete bestehen aus 222 Einzugsgebieten, wobei es sich teilweise um Teileinzugsgebiete der Trainingsgebiete handelt. Die Lage und Größe aller Einzugsgebiete ist in Abb. 5a dargestellt. Die Eigenschaften der Trainingsgebiete sind in Tab. 1 zusammengefasst. Im Rahmen der Modellierung wurden für das Training die Jahre 2000-2004 (5 Jahre) und für die Validierung die Jahre 1965-1999 (35 Jahre) verwendet. Das Modell wurde dabei jeweils mit 
Tagesdaten und 5 Jahren Spin-up gerechnet. Die zur Verfügung stehenden Gebietseigenschaften sind Sandanteil (sand), Tonanteil (ton), Boden-Lagerungsdichte (bd) in $\mathrm{g} / \mathrm{cm}^{3}$, Exposition in Grad, Hangneigung in Grad und Höhe in m. Eine Zusammenfassung des Modellaufbaus und der Inputs ist in Abb. 5b dargestellt.

Neben den Gebietseigenschaften, die in allen FSO-Transferfunktionen (auch mehrmals) beinhaltet sein können, wurden zusätzlich auch die mHMParameter KSat, ThetaS und vGenu_n als mögliche Inputs für die FieldCapTransferfunktion zugelassen. Für beide Transferfunktionen wurde eine CFG erstellt, die neben den Gebietseigenschaften und weiteren Modellparametern die mathematischen Operatoren $+,-, *, /$ beinhaltet, sowie eine Reihe an mathematischen Funktionen: die Exponentialfunktion, der Logarithmus mit Basis 2 oder 10, trigonometrische Funktionen (Sinus, Cosinus, Tangens) sowie deren Arkus- und Hyperbolicusfunktionen, die Betragsfunktion, die Wurzelfunktion und die Potenzfunktion. Aus der Grammatik wurden 45 Mio. unterschiedliche Transferfunktionen erzeugt und ihre Parameterverteilung mittels der Gebietseigenschaften geschätzt. Mit diesem Datensatz wurde für jeden der beiden Parameter ein FSO VAE trainiert.

Als Loss-Funktion für die Optimierung von FSO-mHM verwenden wir eine Kombination (power mean) der zwei Modellgütekriterien NSE und log NSE, die auch schon von Zink et al. (2017) angewandt wurde:

$$
\begin{aligned}
& \text { Loss } \left.=\left(\frac{1}{2} \sum_{i}^{2} \phi_{i}^{p}\right)\right)^{\frac{1}{p}}, \\
& \text { mit } p=6, \phi_{1}=\text { NSE und } \phi_{2}=\log N S E .
\end{aligned}
$$

Mit dieser Loss-Funktion werden Nieder- sowie Hochwassersituationen gleichermaßen in der Zielfunktion berücksichtigt. Zusätzlich zu NSE und log NSE werden die KGE-Ergebnisse aller Modelle berechnet, um eine klare Übersicht der Modellgüte zu geben. Die drei Gütefunktionen sind wie folgt definiert:

$$
\begin{gathered}
N S E=1-\frac{\sum_{t=1}^{T}\left(\widehat{Q}_{t}-Q_{t}\right)}{\sum_{t=1}^{T}\left(Q_{t}-\bar{Q}\right)}, \\
\log N S E=1-\frac{\sum_{t=1}^{T}\left(\log \widehat{Q}_{t}-\log Q_{t}\right)}{\sum_{t=1}^{T}\left(\log Q_{t}-\overline{\log Q}\right)}, \\
K G E=1-\sqrt{(r-1)^{2}+\left(\frac{\sigma_{\widehat{Q}}}{\sigma_{Q}}-1\right)^{2}+\left(\frac{\mu_{\widehat{Q}}}{\mu_{Q}}-1\right)^{2}},
\end{gathered}
$$

wobei $r$ der Pearson Korrelationskoeffizient ist, $\sigma_{\widehat{Q}}$ und $\sigma_{Q}$ die Standardabweichung und $\mu_{\widehat{Q}}$ und $\mu_{Q}$ die Mittelwerte der vorhergesagten und beobachteten Abflüsse sind. Alle drei Gütekriterien haben einen Wertebereich von $[-\infty, 1]$, wobei der Wert 1 eine perfekte Wiederherstellung der Beobachtungen darstellen würde.

Für die Optimierung der numerischen Parameter und der Transferfunktionen aus dem VAE wird der shuffled complex evolution-Algorithmus (SCE, Duan et al. 1992) angewandt. Das Ziel ist dabei die Minimierung der gewichteten Loss-Funktion in 2000 Iterationsschritten.

\section{Ergebnisse}

\subsection{FSO-Transferfunktionen}

Die mHM-FSO-Optimierung an 7 großen Einzugsgebieten ergab neben optimierten numerischen Parametern zwei neue Transferfunktionen für die gesuchten Parameter. Die hydraulische Leitfähigkeit - KSat, welche zuerst mittels Sand- und Tonanteil geschätzt wurde, ist in der FSO-optimierten Version eine Funktion der Boden-Lagerungsdichte (bd):

\section{$\mathrm{KSat}_{\mathrm{FSO}}=-(3.31 \cdot \cosh (\mathrm{bd}))$}

Die FSO-Transferfunktion für den Parameter Feldkapazität (FieldCap)
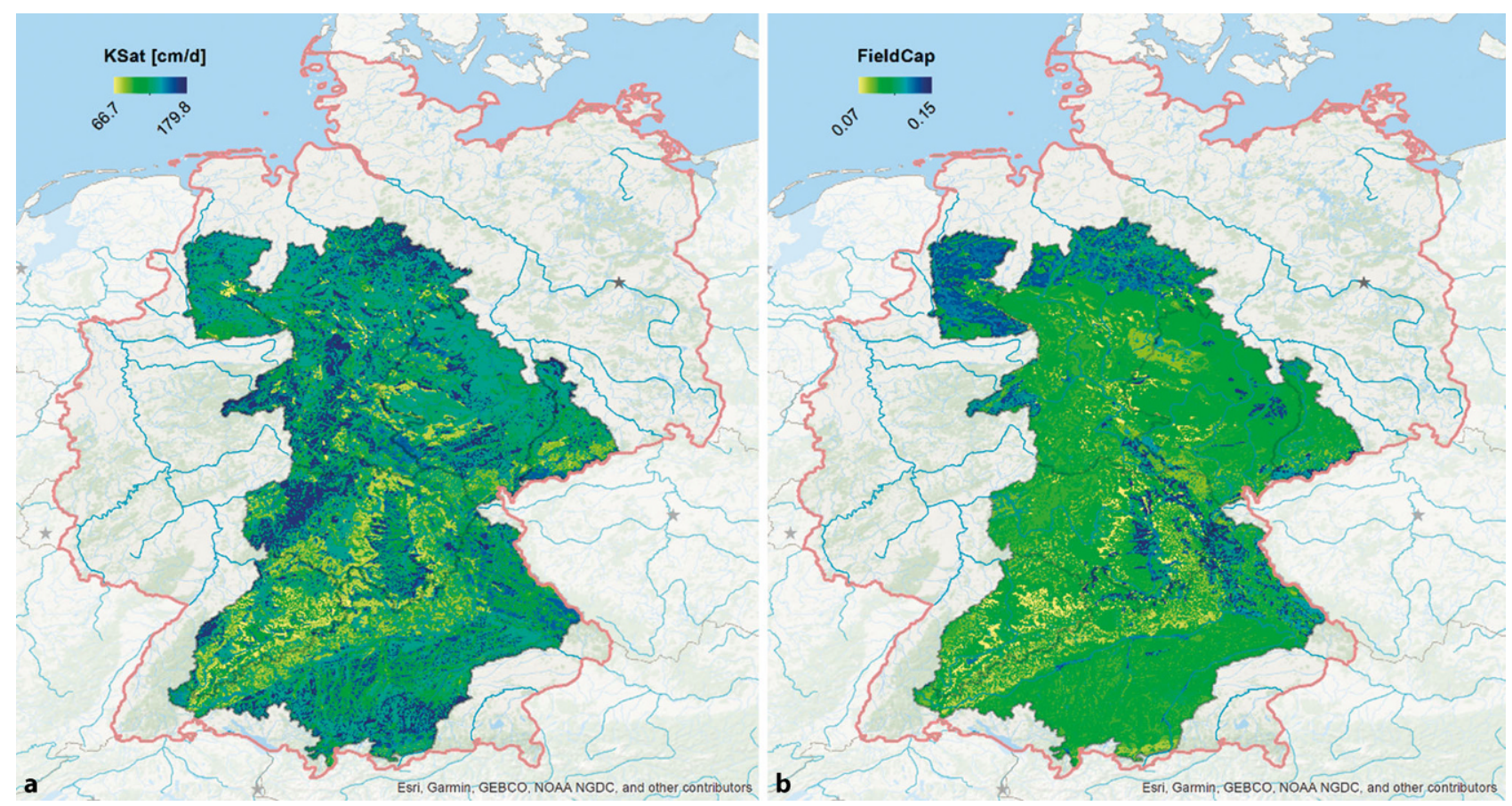

Abb. 6 Parameterfelder der von FSO geschätzten a hydraulischen Leitfähigkeit und b Feldkapazität. Die Werte der Feldkapazität sind als Anteil des gesättigten Bodenwassergehalts angegeben 
Tab. 2 Modellgüte der 7 großen Trainingsgebiete für Trainings- und Validierungsperioden

\begin{tabular}{|c|c|c|c|c|c|c|}
\hline \multirow[t]{2}{*}{ Einzugsgebiet } & \multicolumn{3}{|c|}{ Trainingsperiode } & \multicolumn{3}{|c|}{ Validierungsperiode } \\
\hline & NSE & Log NSE & KGE & NSE & Log NSE & KGE \\
\hline Mulde & 0,80 & 0,75 & 0,71 & 0,60 & 0,66 & 0,59 \\
\hline Ems & 0,75 & 0,70 & 0,75 & 0,75 & 0,72 & 0,73 \\
\hline Neckar & 0,75 & 0,80 & 0,78 & 0,71 & 0,73 & 0,76 \\
\hline Main & 0,82 & 0,77 & 0,84 & 0,78 & 0,77 & 0,86 \\
\hline Saale & 0,68 & 0,72 & 0,82 & 0,54 & 0,48 & 0,75 \\
\hline Weser & 0,79 & 0,79 & 0,85 & 0,80 & 0,77 & 0,85 \\
\hline Donau & 0,80 & 0,79 & 0,89 & 0,73 & 0,71 & 0,81 \\
\hline Median & 0,79 & 0,77 & 0,82 & 0,73 & 0,72 & 0,76 \\
\hline
\end{tabular}

verwendet ähnlich wie die originale mHM-Funktion den Parameter ThetaS (gesättigter Bodenwassergehalt) und KSat, aber nicht den vGenu_n (van-Genuchten-Modell)-Parameter, der in der Originalfunktion zu finden ist. Dafür wird, ähnlich wie bei KSat, eine Funktion der Boden-Lagerungsdichte (bd) verwendet:

FieldCap $_{\mathrm{FSO}}=$

KSat $-(2.781+$ ThetaS $\cdot \cosh (b d))$.
Zugunsten der Lesbarkeit verzichten wir in dieser Darstellung der Transferfunktionen auf die Skalierungskoeffizienten, welche verwendet werden, um die endgültigen Parameterwerte zu berechnen. Die resultierenden Werte für KSat liegen im Intervall $[66,7$, $179,8] \mathrm{cm} /$ Tag, die Werte für FieldCap im Intervall $[0,07,0,15]$. Hier ist nochmals anzumerken, dass sich die in mHM verwendete Feldkapazität nicht auf das gesamte Porenvolumen, sondern nur auf den gesättigten Bodenwas- sergehalt bezieht. Die räumliche Verteilung beider Parameter ist in Abb. 6a, b dargestellt.

\subsection{Vorhersage in den Trainingsgebieten}

Eine Übersicht der Ergebnisse der 7 Trainingsgebiete ist in Tab. 2 dargestellt. Die beiden Gütekriterien der Loss-Funktion, NSE und log NSE, sind mit medianen Werten von 0,79 und 0,77 in der Trainingsperiode ähnlich hoch.
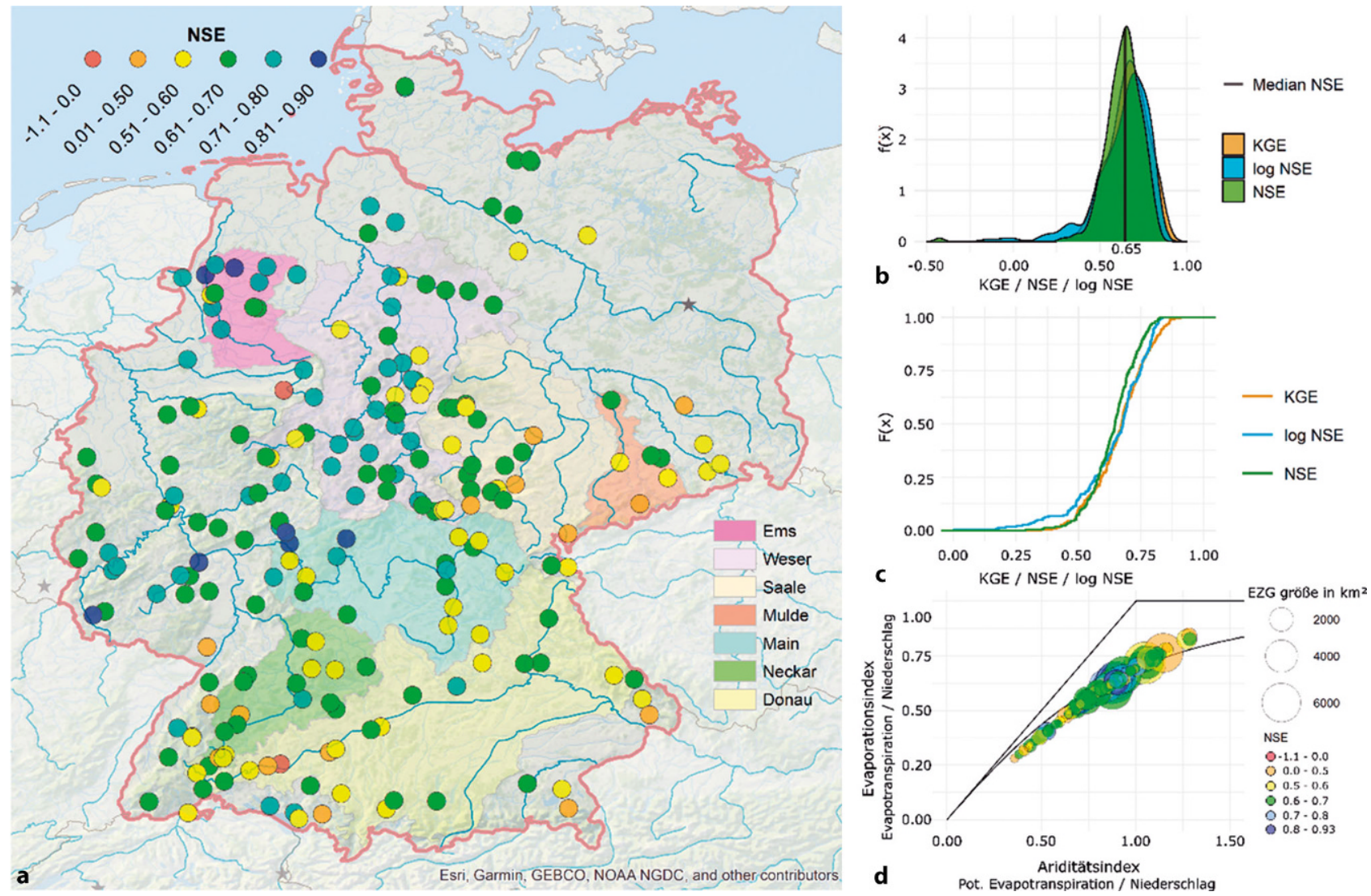

Abb. 7 a NSE-Ergebnisse der Validierungsgebiete, dargestellt als Punkte der Messstationen mit farblicher Kennzeichnung des NSE-Werts. b Verteilungsdichten der Modellgüterkriterien der 222 Validierungsgebiete mit eingezeichnetem medianen NSE-Wert von 0,65. c Kumulative Verteilung der Modellgütekriterien. d Budyko-Kurve (Budyko 1974) der Validierungsgebiete mit Darstellung der Gebietsgröße als Punktgröße und farblich gekennzeichneten NSE-Werten 
Bei beiden ist auch nur eine geringe Reduktion in der Validierungsperiode ersichtlich: 0,06 für den medianen NSE und 0,05 für den medianen log NSE. Während sich in den meisten Gebieten die Modellgüte kaum ändert, ist in den Gebieten Saale und Mulde eine stärkere Reduktion zu erkennen. Die Werte des KGE-Kriteriums, welches allerdings nicht Teil des Optimierungsziels war, sind mit einem Median von 0,82 in der Trainingsperiode und 0,76 in der Validierungsperiode ähnlich hoch.

\subsection{Vorhersage in Validierungsgebieten}

Die Ergebnisse der Anwendung der von FSO geschätzten Transferfunktionen und numerischen mHM-Parametern in den Validierungsgebieten in der Validierungszeitperiode sind in Abb. 7 zusammengefasst. In Abb. 7a sind die NSE-Werte aller Validierungsgebiete und die Position der zugehörigen Messstellen dargestellt. In Abb. 7b, c sind die Verteilungsdichte und die kumulative Verteilungsfunktion von NSE-, log NSEund KGE-Werten der Validierungsgebiete dargestellt. Abb. 7d zeigt die Position der Validierungsgebiete auf der Budyko Kurve (Budyko 1974) sowie die Gebietsgröße und NSE-Werte.

Im Validierungszeitraum zeigen die Validierungsgebiete einen medianen NSE von 0,65 und erreichen somit eine Modellgüte, die nicht sehr viel geringer ist als die der Trainingsgebiete mit einem medianen NSE von 0,73 . Wie auch im Training sind in der Validierung die Verteilungen der weiteren Modellgütekriterien (Abb. 7b, c) sehr ähnlich, mit einem medianen log NSE von 0,68 und einem medianen KGE von 0,67.

Insgesamt haben $17 \quad(7,7 \%)$ der 222 Validierungsgebiete einen NSE von weniger als $0,5,34$ Gebiete $(15,3 \%)$ einen log NSE von weniger als 0,5 und $22(10 \%)$ Gebiete einen KGE von weniger als 0,5. Diese Gebiete befinden sich hauptsächlich im Süden und im Osten Deutschlands und liegen im Mittel signifikant höher als die restlichen Validierungsgebiete. Ein Zusammenhang zwischen der Modellgüte und der Lage der Einzugsgebiete auf der Budyko-Kurve ist hingegen nicht erkennbar und auch nicht statistisch feststellbar (Abb. 7d). Somit sind die Vorhersagen in trockenen (Ariditätsindex $>1$ ) und feuchteren (Ariditätsindex < 1) Gebieten gleichermaßen gut. Des Weiteren weisen die Validierungsgebiete außerhalb der Trainingsgebiete keine niedrigeren
Modellgütewerte auf als die innerhalb der Trainingsgebiete gelegenen Validierungsgebiete.

\section{Diskussion}

In diesem Beitrag präsentieren wir die FSO-Methode für das automatische Schätzen von Transferfunktionen für hydrologische Modelle. Das Erzeugen von räumlich verteilten Modellparametern mittels der geophysikalischen Eigenschaften der Einzugsgebiete kann die physikalische Interpretierbarkeit des Modells erhöhen, erzeugt kontinuierliche Parameterfelder und ermöglicht die Vorhersage in Einzugsgebieten ohne Messstellen. Die FSO-Methode wandelt die Suche nach einer passenden Transferfunktion in ein kontinuierliches Optimierungsproblem um und kann daher mit den üblichen Kalibrierungsmethoden der hydrologischen Modellierung gelöst werden.

In dieser Arbeit wurde die Anwendung von FSO am Beispiel des mHM (Mesocale Hydrological Model) gezeigt. Dabei wurden die Daten von 7 Messstellen über einen Zeitraum von 5 Jahren verwendet, um die Transferfunktionen zweier Parameter, gesättigte hydraulische Leitfähigkeit und Feldkapazität, zu optimieren. Zum Testen der gefunden Transferfunktionen wurden diese in 222 Einzugsgebieten mit Daten aus einem Zeitraum von 35 Jahren validiert. Dabei beinhaltete die Anwendung in den Validierungsgebieten keinerlei Optimierung bzw. Kalibrierung und erreichte dennoch hohe ModellgüteWerte (NSE >0,5) in fast allen Gebieten.

Ein Vorteil der Parameterschätzung mittels Transferfunktion ist die physikalische Interpretierbarkeit der Parameter und der Modellzustände (z.B. Speicher). Hydrologische Modelle wie mHM, welches zur Vorhersage der Bodenfeuchtezustände in Deutschland verwendet wird (Zink et al. 2016; Dürremonitor Deutschland: https://www. ufz.de/index.php?de=37937), sind auch äußert relevant in Situationen, in denen unbeobachtete Komponenten des Wasserkreislaufes geschätzt werden müssen. Das unterscheidet sie somit von rein datengetriebenen Modellierungsansätzen, welche in den letzten Jahren vermehrt zum Einsatz gekommen sind und auch bereits zur Abflussvorhersage in Gebieten ohne Messstellen angewendet wurden (e.g. Kratzert et al. 2019). In einer vorangegangenen Studie (Feigl et al. 2020) konnten wir zeigen, dass FSO auch mit zusätzlichen Beobachtungen der Speicherzustände in den Einzugsgebieten kalibriert werden kann und damit Unsicherheiten in der Vorhersage von Speicherzuständen und Parameterfeldern reduziert werden können. Schlussendlich sehen wir die FSO-Methode als eine Möglichkeit, mit datengetriebenen Methoden hydrologische Modelle weiterzuentwickeln und ihre Vorhersagefähigkeiten und physikalische Interpretierbarkeit zu verbessern.

Das hier präsentierte Fallbeispiel zeigt sehr gut die Stärken der FSOMethode, gleichzeitig aber auch die Limitation auf. Aufgrund der Tatsache, dass höher gelegene Gebiete in Südund Ostdeutschland geringere Modellgütewerte in der Validierung aufweisen, können wir annehmen, dass höher liegende Gebiete nicht ausreichend in den Trainingsgebieten repräsentiert waren. An diesem Beispiel ist gut erkennbar, dass die Wahl der Gebiete essenziell für repräsentierbare Transferfunktionen ist. Neben der Wahl der Trainingsgebiete ist auch die Auswahl der geophysikalischen Gebietseigenschaften wichtig für die Anwendung von FSO. Nur wenn relevante verteilte Informationen für Parameter vorhanden sind, können die dazugehörigen Transferfunktionen geschätzt werden. Dabei ist anzumerken, dass durch die häufig hohe Korrelation von Gebietseigenschaften auch die Möglichkeit besteht, dass fehlende Informationen durch andere Gebietseigenschaften approximiert werden können. Des Weiteren ist die Interpretation der resultierenden Parameterfelder nicht einfach, da die hier geschätzten Bodeneigenschaften stark skalenabhängig sind und in einer $100 \times 100$-m- oder $4 \times 4-\mathrm{km}$ Gridzelle vollkommen andere Wertebereiche und Interpretationen aufweisen können als z.B. Laborproben (Hrachowitz et al. 2013). Zusätzlich ist die physikalische Interpretierbarkeit der resultierenden Parameter vom jeweiligen Modell abhängig. Potenziell können auch verwendbare Transferfunktionen bei konzeptionellen Modellen gefunden werden.

Die Anwendung verteilter hydrologischer Modelle in Gebieten ohne Messstellen ist aufgrund der bis $\mathrm{zu}$ einem gewissen Ausmaß nötigen Parameterkalibrierung eingeschränkt (Beven 2001; Kirchner 2006). Diese Problematik beschäftigt die Hydrologie sowie die landeskulturelle Wasserwirtschaft 
Hier steht eine Anzeige.

Springer 
Hier steht eine Anzeige.

Springer 
seit mehreren Dekaden (Godina und Blöschl 2006; Hrachowitz et al. 2013). Im Gegensatz zu anderen Methoden der Regionalisierung, welche häufig auf der Ähnlichkeit zu anderen Gebieten in der Region basieren (Razavi und Coulibaly 2013), erlaubt die Regionalisierung mittels Transferfunktionen eine von anderen Gebieten unabhängige Anwendung. Mit FSO ist zum ersten Mal eine objektive, datengetriebene Methode vorhanden, mit der Transferfunktionen geschätzt werden können. Damit ist eine der größten Restriktionen der Anwendung der MPR-Methode, die Wahl einer geeigneten Transferfunktion (Samaniego et al. 2017), gelöst.

\section{Schlussfolgerungen und Ausblick}

Parameter-Transferfunktionen haben das Potenzial, bestehende hydrologische Modelle zu verbessern, eine Übertragbarkeit auf unbeobachtete Gebiete zu gewährleisten und die physikalische Interpretierbarkeit der Parameter und Modellspeicher zu erhöhen. FSO ist eine geeignete Methode zum Schätzen von Transferfunktionen und ist auf alle verteilten Modelle anwendbar. Zur Weiterentwicklung dieses Ansatzes sind vor allem die Anwendung mit weiteren prozessbasierten verteilten Modellen, die Anwendung in größeren Regionen mit unterschiedlicheren Klimabedingungen sowie das Generieren zusätzlicher verteilter Gebietseigenschaften als nächste Schritte geplant. Vor allem Informationen $\mathrm{zu}$ Vegetation und Verdunstungseigenschaften sind wichtig für hydrologische Prozesse und können potenzielle Inputs für eine Reihe von Transferfunktionen vieler Modelle sein. Schlussendlich ist auch eine Implementierung von FSO als eigenständiges Softwarepaket geplant, welches die Anwendung auf beliebigen Modellen vereinfachen soll.

Funding Diese Studie wurde vom Österreichischen Wissenschaftsfonds FWF, Projektnummer P 31213 finanziert. Alle Berechnungen wurden auf dem Vienna Scientific Cluster (VSC) durchgeführt.

Funding Open access funding provided by University of Natural Resources and Life Sciences Vienna (BOKU).

Open Access Dieser Artikel wird unter der Creative Commons Namensnen- nung 4.0 International Lizenz veröffentlicht, welche die Nutzung, Vervielfältigung, Bearbeitung, Verbreitung und Wiedergabe in jeglichem Medium und Format erlaubt, sofern Sie den/die ursprünglichen Autor(en) und die Quelle ordnungsgemäß nennen, einen Link zur Creative Commons Lizenz beifügen und angeben, ob Änderungen vorgenommen wurden.

Die in diesem Artikel enthaltenen Bilder und sonstiges Drittmaterial unterliegen ebenfalls der genannten Creative Commons Lizenz, sofern sich aus der Abbildungslegende nichts anderes ergibt. Sofern das betreffende Material nicht unter der genannten Creative Commons Lizenz steht und die betreffende Handlung nicht nach gesetzlichen Vorschriften erlaubt ist, ist für die oben aufgeführten Weiterverwendungen des Materials die Einwilligung des jeweiligen Rechteinhabers einzuholen.

Weitere Details zur Lizenz entnehmen Sie bitte der Lizenzinformation auf http://creativecommons.org/licenses/ by/4.0/deed.de.

Literatur

Abdulla, F. A., \& Lettenmaier, D. P. (1997): Development of regional parameter estimation equations for a macroscale hydrologic model. Journal of Hydrology, 197(1-4), 230-257. https:// doi.org/10.1016/S0022-1694(96)03262-3

Bai, S., Kolter, J. Z., \& Koltun, V. (2018): An Empirical Evaluation of Generic Convolutional and Recurrent Networks for Sequence Modeling. http://arxiv.org/abs/1803.01271. Zugegriffen: 18.01.2021

Bergstrom, S. (1995): The HBV Model. In Singh, V.P. (Hrgs) Computer Models of Watershed Hydrology. Water Resources Publications, Highlands Ranch, CO, pp. 443-476.

Beven, K. (2001): How far can we go in distributed hydrological modelling? Hydrology and Earth System Sciences, 5(1), 1-12. https://doi org/10.5194/hess-5-1-2001

Beven, Keith. (2006): A manifesto for the equifinality thesis. Journal of Hydrology, 320(1-2), 18-36 https://doi.org/10.1016/JJHYDROL. 2005.07.007

Blöschl, G., Bierkens, M. F. P., Chambel, A., Cudennec, C., Destouni, G., Fiori, A., Kirchner, J. W., McDonnell, J. J., Savenije, H. H. G., Sivapalan, M., Stumpp, C., Toth, E., Volpi, E., Carr, G. Lupton, C., Salinas, J., Széles, B., Viglione, A. Aksoy, H., Allen, S. T., Amin, A., Andréassian, V., Arheimer, B., Aryal, S., Baker, V., Bardsley, E., Barendrecht, M. H., Bartosova, A., Batelaan, O., Berghuijs, W. R., Beven, K., Blume, T. Bogaard, T., Borges de Amorim, P., Böttcher, M. E., Boulet, G., Breinl, K., Brilly, M., Brocca, L., Buytaert, W., Castellarin, A., Castelletti, A. Chen, X., Chen, Y., Chen, Y., Chifflard, P, Claps,

P., Clark, M., Collins, A., Croke, B., Dathe, A.,
David, P. C., de Barros, F. P. J., de Rooij, G., di Baldassarre, G., Driscoll, J. M., Düthmann, D., Dwivedi, R., Eris, E., Farmer, W. H., Feiccabrino, J., Ferguson, G., Ferrari, E., Ferraris, S., Fersch, B., Finger, D., Foglia, L., Fowler, K., Gartsman, B., Gascoin, S., Gaume, E., Gelfan, A., Geris, J. Gharari, S., Gleeson, T., Glendell, M., Gonzalez Bevacqua, A., González-Dugo, M. P., Grimaldi, S., Gupta, A. B., Guse, B., Han, D., Hannah, D., Harpold, A., Haun, S., Heal, K., Helfricht, K., Herrnegger, M., Hipsey, M., Hlaváčiková, H., Hohmann, C., Holko, L., Hopkinson, C., Hrachowitz, M., Illangasekare, T. H., Inam, A. Innocente, C., Istanbulluoglu, E., Jarihani, B., Kalantari, Z., Kalvans, A., Khanal, S., Khatami, S., Kiesel, J., Kirkby, M., Knoben, W., Kochanek, K., Kohnova, S., Kolechkina, A., Krause, S., Kreamer, D., Kreibich, H., Kunstmann, H., Lange, H., Liberato, M. L. R., Lindquist, E., Link, T., Liu, J., Loucks, D. P., Luce, C., Mahé, G., Makarieva, O., Malard, J., Mashtayeva, S., Maskey, S., Mas-Pla, J., Mavrova-Guirguinova, M., Mazzoleni, M., Mernild, S., Misstear, B. D. Montanari, A., Müller-Thomy, H., Nabizadeh, A., Nardi, F., Neal, C., Nesterova, N., Nurtaev, B., Odongo, V., Panda, S., Pande, S., Pang, Z. Papacharalampous, G., Perrin, C., Pfister, L. Pimentel, R., Polo, M. J., Post, D., Prieto Sierra, C., Ramos, M. H., Renner, M., Reynolds, J. E., Ridolfi, E., Rigon, R., Riva, M., Robertson, D., Rosso, R., Roy, T., Sá, J. H. M., Salvadori, G., Sandells, M., Schaefli, B., Schumann, A. Scolobig, A., Seibert, J., Servat, E., Shafiei, M. Sharma, A., Sidibe, M., Sidle, R. C., Skaugen, T. Smith, H., Spiessl, S. M., Stein, L., Steinsland, I., Strasser, U., Su, B., Szolgay, J., Tarboton, D.
Tauro, F., Thirel, G., Tian, F., Tong, R., Tussupova, K., Tyralis, H., Uijlenhoet, R., van Beek, R, van der Ent, $R$. J., van der Ploeg, M., van Loon, A. F., van Meerveld, I., van Nooijen, R. van Oel, P. R., Vidal, J. P., von Freyberg, J., Vorogushyn, S., Wachniew, P., Wade, A., Ward, P., Westerberg, I., White, C., Wood, E. F, Woods, R., Xu, Z., Yilmaz, K. K., \& Zhang, Y. (2019): Twenty-three unsolved problems in hydrology (UPH) - a community perspective. Hydrological Sciences Journal, 64(10), 1141-1158. https://doi. org/10.1080/02626667.2019.1620507

Bongard, J., \& Lipson, H. (2007): Automated reverse engineering of nonlinear dynamical systems. Proceedings of the National Academy of Sciences of the United States of America 104(24), 9943-9948. https://doi.org/10.1073/ pnas.0609476104

Budyko, M. I. (1974): Climate and life. Academic Press. https://agris.fao.org/agris-search/search. do?recordID=US201300514816

Buytaert, W., \& Beven, K. (2009): Regionalization as a learning process. Water Resources Research, 45(11). https://doi.org/10.1029/2008 WR007359

Clark, M. P., Bierkens, M. F. P., Samaniego, L., Woods, R. A., Uijlenhoet, R., Bennett, K. E. Pauwels, V. R. N., Cai, X., Wood, A. W., \& PetersLidard, C. D. (2017): The evolution of processbased hydrologic models: historical challenges and the collective quest for physical realism. Hydrology and Earth System Sciences, 21(7), 3427-3440. https://doi.org/10.5194/hess-213427-2017

Clark, M. P., Schaefli, B., Schymanski, S. J., Samaniego, L., Luce, C. H., Jackson, B. M., Freer, 
J. E., Arnold, J. R., Moore, R. D., Istanbulluoglu, E., \& Ceola, S. (2016): Improving the theoretica underpinnings of process-based hydrologic models. Water Resources Research, 52(3), 2350-2365. https://doi.org/10.1002/2015WR017910@10. 1002/(ISSN) 1944-9208.COMHES

Cornforth, T. W., \& Lipson, H. (2015): A hybrid evolutionary algorithm for the symbolic modeling of multiple-time-scale dynamical systems Evolutionary Intelligence, 8(4), 149-164. https:// doi.org/10.1007/s12065-015-0126-x

Cosby, B. J., Hornberger, G. M., Clapp, R. B., \& Ginn, T. R. (1984): A Statistical Exploration of the Relationships of Soil Moisture Characteristics to the Physical Properties of Soils. Water Resources Research, 20(6), 682-690. https://doi. $\mathrm{org} / 10.1029 /$ WR020i006p00682

Le Cun, Y., \& Fogelman-Soulié, F. (1987): Modèles connexionnistes de l'apprentissage. Intellectica. Revue de l'Association Pour La Recherche Cognitive, 2(1), 114-143. https://doi.org/10. 3406/intel.1987.1804

Devia, G. K., \& Ganasri, B. P. (2015): A Review on Hydrological Models. Aquatic Procedia, 4 1001-1007. https://doi.org/10.1016/J.AQPRO. 2015.02.126

Duan, Q., Sorooshian, S., \& Gupta, V. (1992): Effective and efficient global optimization for conceptual rainfall-runoff models. Water $\mathrm{Re}$ sources Research, 28(4), 1015-1031. https://doi. org/10.1029/91WR02985

Feigl, M., Herrnegger, M., Klotz, D., \& Schulz, K. (2020): Function Space Optimization: A Symbolic Regression Method for Estimating Paramete Transfer Functions for Hydrological Models. Water Resources Research, 56(10). https://doi.org/ 10.1029/2020WR027385

van Genuchten, M. T. (1980): A Closed-form Equation for Predicting the Hydraulic Conductivity of Unsaturated Soils. Soil Science Society of America Journal, 44(5), 892-898. https://doi. org/10.2136/sssaj1980.03615995004400050002x Godina, R., \& Blöschl, G. (2006): Aufgaben und Methoden der hydrologischen Regionalisierung In Methoden der hydrologischen Regionalisierung (Vol. 197, pp. 7-20).

Gupta, H. V., Kling, H., Yilmaz, K. K., \& Martinez, G. F. (2009): Decomposition of the mean squared error and NSE performance criteria: Implications for improving hydrological modelling. Journal of Hydrology, 377(1-2), 80-91. https:// doi.org/10.1016/j.jhydrol.2009.08.003

Hattermann F.F., Krysanova V., Gosling S.N. Dankers R., Daggupati P., Donnelly C., Flörke M., Huang S., Motovilov Y., Buda S., Yang T., Müller C., Leng G., Tang Q., Portmann F.T. Hagemann S., Gerten D., WadaY., Masaki Y., Alemayehu T., Satoh Y., Samaniego L. (2017): Cross-scale intercomparison of climate change impacts simulated by regional and global hydrological models in eleven large river basins Climatic Change, 141(3), 561-576. https://doi org/10.1007/s10584-016-1829-4

Hochreiter, S., \& Schmidhuber, J. (1997): Long Short-Term Memory. Neural Computation, 9(8), 1735-1780. https://doi.org/10.1162/neco.1997. 9.8 .1735

Holland, J. (1975): Adaptation in natural and artificial systems. an introductory analysis with applications to biology, control and artificia intelligence. Ann Arbor: University of Michigan Press, 1975. http://adsabs.harvard.edu/abs/ 1975anas.book .....H

Höllering, S., Wienhöfer, J., Ihringer, J., Samaniego, L., \& Zehe, E. (2018): Regional analysis of parameter sensitivity for simulation of streamflow and hydrological fingerprints. Hydrol. Earth Syst. Sci, 22, 203-220. https://doi.org/10.5194/ hess-22-203-2018

Hrachowitz, M., Savenije, H.H.G., Blöschl, G., McDonnell, J.J., Sivapalan, M., Pomeroy, J.W., Arheimerh, B., Blume, T., Clark, M.P., Ehret, U.,
Fenicia, F., Freer, J.E., Gelfan, A., Gupta, H.V., Hughes, D.A., Hut, R.W., Montanari, A., Pande, S., Tetzlaff, D., Troch, P.A., Uhlenbrook, S., Wagener, T.,Winsemius, H.C., Woods, R.A., Zehe, E., Cudennec, C. (2013): A decade of Predictions in Ungauged Basins (PUB) - a review. Hydrological Sciences Journal, 58(6), 1198-1255. https:// doi.org/10.1080/02626667.2013.803183 Hundecha, Y., \& Bárdossy, A. (2004): Modeling of the effect of land use changes on the runoff generation of a river basin through parameter regionalization of a watershed model. Journal of Hydrology, 292(1-4), 281-295. https://doi.org/ 10.1016/J.JHYDROL.2004.01.002

Kay, A. L., Rudd, A. C., Davies, H. N., Kendon, E. J., \& Jones, R. G. (2015): Use of very high resolution climate model data for hydrological modelling: baseline performance and future flood changes. Climatic Change, 133(2), 193-208. https://doi.org/10.1007/s10584-015-1455-6

Kennedy, J., \& Eberhart, R. (1995): Particle swarm optimization. Proceedings of ICNN'95International Conference on Neural Networks, 4 1942-1948. https://doi.org/10.1109/ICNN.1995. 488968

Kingma, D. P., \& Welling, M. (2013): Auto-Encoding Variational Bayes. http://arxiv.org/abs/ 1312.6114. Zugegriffen: 22.10.2019

Kirchner, J. W. (2006): Getting the right answers for the right reasons: Linking measurements, analyses, and models to advance the science of hydrology. Water Resources Research, 42(3). https://doi.org/10.1029/2005WR004362

Klambauer, G., Unterthiner, T., Mayr, A., \& Hochreiter, S. (2017): Self-Normalizing Neural Networks. http://arxiv.org/abs/1706.02515. Zugegriffen: 23.10.2019

Klotz, Daniel. (2020): Systematic estimation of transferfunctions for the parameterization of spatially distributed rainfall-runoff models [University of Natural Resources and Life Sciences, Vienna]. https://permalink.obvsg.at/ AC16121579

Klotz, D., Herrnegger, M., \& Schulz, K. (2017): Symbolic Regression for the Estimation of Transfer Functions of Hydrological Models. Water Re sources Research, 53(11), 9402-9423. https://doi. org/10.1002/2017WR021253

Knuth, D. E. (1965): On the translation of languages from left to right. Information and Control, 8(6), 607-639. https://doi.org/10.1016/ S0019-9958(65)90426-2

Koza, J. R. (1992): Genetic programming: on the programming of computers by means of natural selection. MIT Press. https://books.google at/books/about/Genetic_Programming.html? id=Bhtxo60BV0EC\&redir_esc=y. Zugegriffen 08.03 .2021

Kratzert, F., Klotz, D., Shalev, G., Klambauer, G., Hochreiter, S., \& Nearing, G. (2019): Towards learning universal, regional, and local hydrological behaviors via machine learning applied to large-sample datasets. Hydrology and Earth System Sciences, 23(12), 5089-5110. https://doi. org/10.5194/hess-23-5089-2019

Kumar, R., Samaniego, L., \& Attinger, S. (2013a) Implications of distributed hydrologic model parameterization on water fluxes at multiple scales and locations. Water Resources Research, 49(1), 360-379. https://doi.org/10.1029/ 2012WR012195

Kumar, R., Samaniego, L., \& Attinger, S. (2013b) Implications of distributed hydrologic model parameterization on water fluxes at multiple scales and locations. Water Resources Research, 49(1), 360-379. https://doi.org/10.1029/ 2012WR012195

Van Looy, K., Bouma, J., Herbst, M., Koestel, J. Minasny, B., Mishra, U., Montzka, C., Nemes, A., Pachepsky, Y. A., Padarian, J., Schaap, M. G.,

Tóth, B., Verhoef, A., Vanderborght, J., Ploeg, M.

J., Weihermüller, L., Zacharias, S., Zhang, Y., \&
Vereecken, H. (2017): Pedotransfer Functions in Earth System Science: Challenges and Perspectives. Reviews of Geophysics, 55(4), 1199-1256. https://doi.org/10.1002/2017RG000581

Lu, S., Zhu, Y., Zhang, W., Wang, J., \& Yu, Y. (2018): Neural Text Generation: Past, Presen and Beyond. http://arxiv.org/abs/1803.07133. Zugegriffen: 06.11.2019

Mikolov, T., Chen, K., Corrado, G., \& Dean, J. (2013): Efficient Estimation of Word Representations in Vector Space. http://arxiv.org/abs/1301. 3781. Zugegriffen: 28.10.2019

Nash, J. E., \& Sutcliffe, J. V. (1970): River flow forecasting through conceptual models part I-A discussion of principles. Journal of Hydrology, 10(3), 282-290. https://doi.org/10.1016/0022 1694(70)90255-6

Parajka, J., Merz, R., \& Blöschl, G. (2005): A comparison of regionalisation methods for catchment model parameters. Hydrology and Earth System Sciences Discussions, 9(3), 157-171. https://hal.archives- ouvertes.fr/hal-00304814/ Rakovec, O., Kumar, R., Mai, J., Cuntz, M., Thober, S., Zink, M. Attinger, S., Schäfer, D., Schrön, M., Samaniego, Lo, Rakovec, O, Kumar, R., Mai, J., Cuntz, M., Thober, S., Zink, M., Attinger, S., Schäfer, D., Schrön, M., \& Samaniego, L. (2016): Multiscale and Multivariate Evaluation of Water Fluxes and States over European River Basins. Journal of Hydrometeorology, 17(1) 287-307. https://doi.org/10.1175/JHM-D- 150054.1

Razavi, T., \& Coulibaly, P. (2013): Streamflow Prediction in Ungauged Basins: Review of Regionalization Methods. Journal of Hydrologic Engineering, 18(8), 958-975. https://doi.org/10.1061/ (asce)he.1943-5584.0000690

Samaniego, L., \& Bárdossy, A. (2005): Robust parametric models of runoff characteristics at the mesoscale. Journal of Hydrology, 303(1-4), 136-151. https://doi.org/10.1016/j.jhydrol.2004. 08.022

Samaniego, L., Kaluza, M., Kumar, R., Rakovec, O., Schüler, L., Schweppe, R., Shrestha, P K., Thober, S., \& Attinger, S. (2019): mesoscale Hydrologic Model. https://doi.org/10.5281/ ZENODO.3239055

Samaniego, L., Kumar, R., \& Attinger, S. (2010): Multiscale parameter regionalization of a gridbased hydrologic model at the mesoscale. $\mathrm{Wa}$ ter Resources Research, 46(5). https://doi.org/10. 1029/2008WR007327

Samaniego, L., Kumar, R., Thober, S., Rakovec, O., Zink, M., Wanders, N., Eisner, S., Mülle Schmied, H., Sutanudjaja, E. H., Warrach-Sagi, K., \& Attinger, S. (2017): Toward seamless hydrologic predictions across spatial scales. Hydrology and Earth System Sciences, 21(9), 4323-4346. https://doi.org/10.5194/hess-21-4323-2017 Schmidt, M., \& Lipson, H. (2009): Distilling free-form natural laws from experimental data. Science (New York, N.Y.), 324(5923), 81-85. https://doi.org/10.1126/science.1165893

Srivastava, S., Shukla, A., \& Tiwari, R. (2018): Machine Translation: From Statistical to modern Deep-learning practices. http://arxiv.org/ abs/1812.04238. Zugegriffen: 06.11.2019

Thober, S., Cuntz, M., Kelbling, M., Kumar, R. Mai, J., \& Samaniego, L. (2019): The multiscale routing model mRM v1.0: Simple river routing at resolutions from 1 to $50 \mathrm{~km}$. Geoscientific Mode Development, 12(6), 2501-2521. https://doi.org/ 10.5194/gmd-12-2501-2019

Tolson, B. A., \& Shoemaker, C. A. (2007): Dynamically dimensioned search algorithm for computationally efficient watershed model calibration. Water Resources Research, 43(1). https://doi. org/10.1029/2005WR004723

White, B. W., \& Rosenblatt, F. (1963): Principles of Neurodynamics: Perceptrons and the Theory of Brain Mechanisms. The American Journal of 


\section{Originalarbeit}

Psychology (Vol. 76, Issue 4, p. 705). https://doi. org/10.2307/1419730

Wijesekara, G. N., Gupta, A., Valeo, C., Hasbani, J.-G., Qiao, Y., Delaney, P., \& Marceau, D. J. (2012): Assessing the impact of future land-use changes on hydrological processes in the Elbow River watershed in southern Alberta, Canada. Journal of Hydrology, 412-413, 220-232. https:// doi.org/10.1016/J.JHYDROL.2011.04.018

Yang, Z., Dai, Z., Yang, Y., Carbonell, J., Salakhutdinov, R., \& Le, Q. V. (2019): XLNet: Generalized Autoregressive Pretraining for Langu- age Understanding http://arxivorg/abs/1906. 08237. Zugegriffen: 06.11.2019

Zacharias, S., \& Wessolek, G. (2007): Excluding Organic Matter Content from Pedotransfer Predictors of Soil Water Retention. Soil Science Society of America Journal, 71(1), 43-50. https:// doi.org/10.2136/sssaj2006.0098

Zink, M., Kumar, R., Cuntz, M., \& Samaniego, L. (2017): A high-resolution dataset of water fluxes and states for Germany accounting for parametric uncertainty. Hydrology and Earth System Sciences, 21(3), 1769-1790. https://doi.org/ 10.5194/hess-21-1769-2017
Zink, M., Samaniego, L., Kumar, R., Thober, S., Mai, J., Schafer, D., \& Marx, A. (2016): The German drought monitor. Environmental Research Letters, 11(7), 074002. https://doi.org/10.1088/ $1748-9326 / 11 / 7 / 074002$

Hinweis des Verlags Der Verlag bleibt in Hinblick auf geografische Zuordnungen und Gebietsbezeichnungen in veröffentlichten Karten und Institutsadressen neutral. 